Alaska Division of Geological \& Geophysical Surveys

PRELIMINARY INTERPRETIVE REPORT 2005-5

\title{
Principal facts for gravity data collected in the northern Susitna Basin area, southcentral Alaska
}

\author{
by \\ John F. Meyer, Jr.
}

August 2005

THIS REPORT HAS NOT BEEN REVIEWED FOR TECHNICAL CONTENT (EXCEPT AS NOTED IN TEXT) OR FOR CONFORMITY TO THE EDITORIAL STANDARDS OF DGGS.

\author{
Released by \\ STATE OF ALASKA \\ DEPARTMENT OF NATURAL RESOURCES \\ Division of Geological \& Geophysical Surveys \\ 3354 College Rd. \\ Fairbanks, Alaska 99709-3707
}





\title{
Principal facts for gravity data collected in the northern Susitna Basin area, southcentral Alaska
}

\author{
by \\ John F. Meyer, Jr. \\ Alaska Division of Oil \& Gas, 550 W $7^{\text {th }}$ Avenue, Suite 800, Anchorage, AK 99501
}

\section{INTRODUCTION}

In order to help stimulate interest in the Susitna basin for petroleum exploration, the Division of Oil and Gas (DO\&G) has been collecting regional gravity data throughout the area to complement and extend the gravity data that is currently available from the U.S. Geological Survey (USGS). During the summer of 2000, DO\&G collected readings from 120 gravity stations along profiles in the southern portion of the basin (Meyer and Boggess, 2003). The current project was designed to obtain data from 90 additional gravity stations to extend these profiles and create new profiles in the northern portion of the basin. The gravity stations collected are primarily located in the Talkeetna Quadrangle and the northwest corner of the Tyonek Quadrangle 1:250,000-scale USGS topographic maps. The study area is bounded approximately by $61^{\circ} 50^{\prime}$ to $62^{\circ} 30^{\prime} \mathrm{N}$ latitude and $151^{\circ} 00^{\prime}$ to $152^{\circ} 30^{\prime} \mathrm{W}$ longitude. Figure 1 is a map of the Susitna basin showing the newly collected gravity stations in addition to the previously collected gravity stations that are currently available from the USGS.

The Susitna basin is a topographic lowland encompassing an area of approximately 4,700 square miles, bordered by the Alaska Range on the north, the Talkeetna Mountains on the east, the Tordrillo Mountains on the west and the Cook Inlet basin on the south. The Susitna basin is considered a northern extension of the Cook Inlet basin, separated from it by the Castle Mountain fault, a major regional structural feature of southcentral Alaska.

The depositional history of the region started during the late Paleozoic and early Triassic when marine sediments were deposited throughout the area. This was followed by a time of deformation and uplift during the late Triassic, which resulted in the deposition of sediments in the Cook Inlet basin to the south. Uplift and erosion of the Alaska Range during the Cretaceous through Tertiary provided the material for a thick sequence of continental shelf deposits throughout the region that were composed of fine-grained sediments rich in organic material. During the Tertiary period, a repetitive cycle of vegetative growth and sediment deposition occurred, depositing sediments along with conglomerates, sands, and clays that created numerous peat layers that were buried, producing the present-day coal formations. The adjacent sands and gravels deposited in the region have become potential reservoirs for oil and gas in the area (Selkregg, 1974; Tim Ryherd, oral commun., 2003). Based on scattered well data in the southern portion of the basin, including the Farms Red Shirt Lake \#1 and Inlet Oil Fish Creek \#1 wells, the region is believed to be underlain by granitic basement rock that is continuous with the Tertiary-Cretaceous age granitic batholith of the Talkeetna Mountains (Turner and Wescott, 1982). 


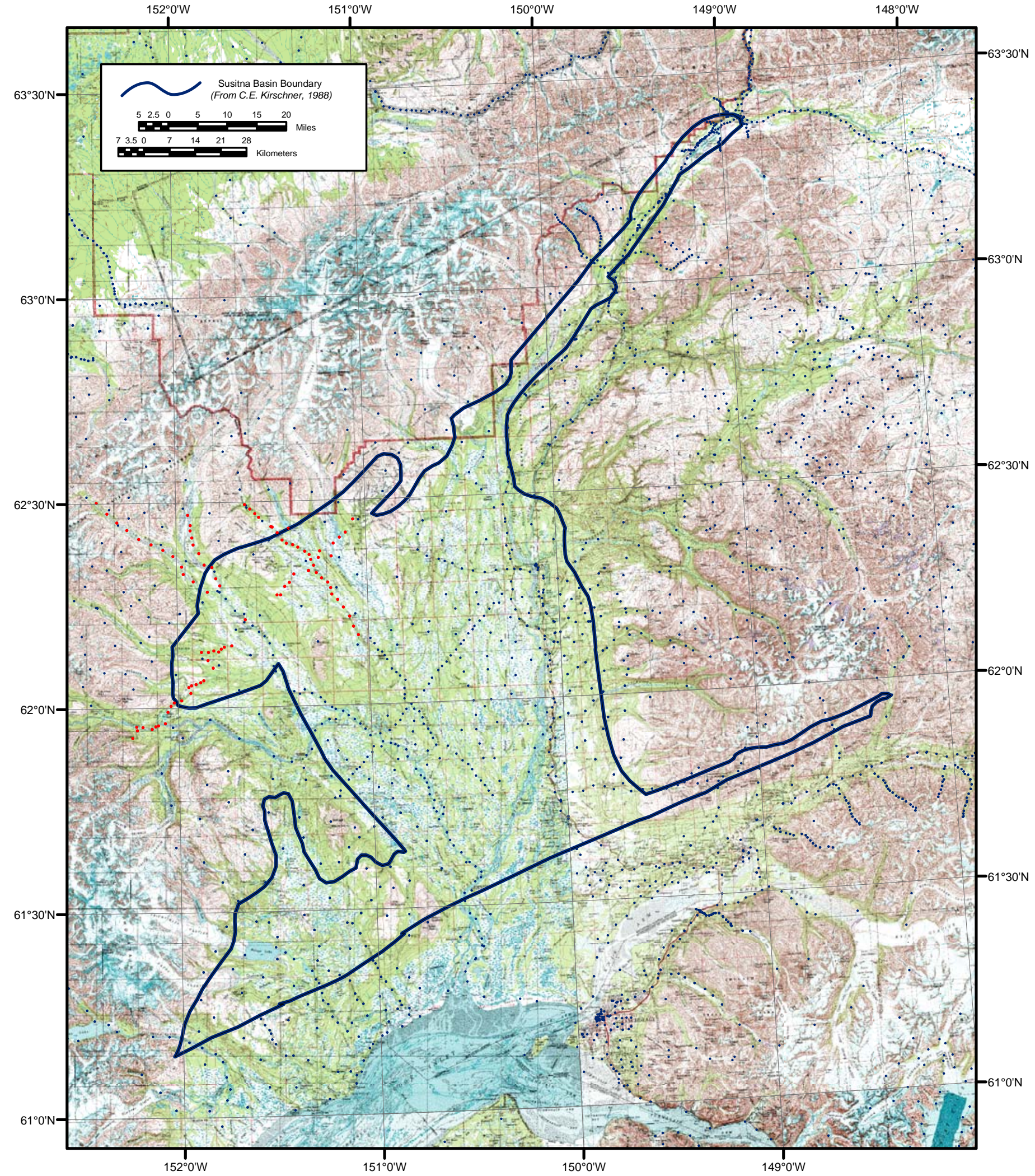

Figure 1. Index map of the study area showing the locations of the gravity data collected for this study (in red). The previously collected data is shown in black. The map base is a composite of USGS 1:250,000-scale topographic maps. 
The structural style of the basin is a combination of graben and half-graben basement faulting with Tertiary sedimentary fill consisting of some of the same formations as found in the Cook Inlet basin. The sedimentary section ranges from about 2,000 feet thick just north of the Castle Mountain Fault to more than 13,000 feet in the center of the basin, while south of the fault it is estimated to be at least 20,000 feet thick (Maynard, 1987; Ryherd, oral commun., 2003). The Eocene-age West Foreland Formation and Oligocene age Hemlock Conglomerate reservoir rocks that are found to the south in the Cook Inlet basin appear to be missing in this basin. The presence of dry gas source rocks in the region, similar to those found in the Cook Inlet basin, and the apparent absence of equivalent oil-prone source rocks indicate that the potential for finding gas in the basin is much greater than for finding oil within the Tertiary section. Coal seams are thick and numerous in parts of the basin, and provide targets for methane drainage drilling as well as a source for gas to charge conventional sandstone reservoirs.

The Susitna basin has not been extensively explored, although a number of oil and gas exploration wells and core tests have been drilled in the region. All of these wells were plugged and abandoned as dry holes, although some did have minor gas shows. There were also prominent coal beds in the lower part of some of the wells, suggesting a correlation with the coal-bearing formations in the Cook Inlet basin that produce natural gas. Taking this into account, the petroleum potential of the basin is thought to be low to moderate (Ryherd, oral commun., 2003).

\section{GRAVITY-DATA ACQUISITION AND REDUCTION}

A LaCoste and Romberg gravity meter (G507) was used to collect the new gravity station data. Conversion of the meter readings to milligals was made using factory calibration constants and a calibration factor determined by Dave Barnes of the USGS. During the field surveys, the gravity meter appeared to function properly, and an average drift of .008 mgal/day with a maximum drift of $0.1 \mathrm{mgal} /$ day indicates there were no apparent tares in the data. The observed gravity values were based on an assumed linear drift between base station readings throughout the day.

Datum control for all of the gravity values was provided by the USGS Alaskan Gravity Base Station Network (Barnes, 1968; 1972) and was adjusted to the new absolute datum of the International Gravity Standardization Net 1971 (Morelli and others, 1974). A second-order base station, CHLK, was also created at Cabin 1 of the Chelatna Lake Lodge to replace the old CHEL base station that was destroyed during construction. The observed gravity of the CHLK station was calculated based on multiple ties to the established TLKM base station. For the duration of the survey, this new base station was reoccupied seven times with survey loops limited to 8 hours or less.

Horizontal control was obtained using a Trimble GeoExplorer 3 handheld Global Positioning System (GPS) unit and 1:63,360-scale USGS topographic maps. The station sites were located on USGS topographic maps in the field and digitized for comparison to the reduced GPS data. The GPS locations were processed using the Trimble GPS Pathfinder Office software and base station data obtained from the NOAA Continuously Operating Reference Stations (CORS) in Talkeetna and Anchorage. Standard processing techniques were applied to the GPS 
data by averaging the corrected data after applying differential corrections and selecting the bestcorrected locations within the 68 percent confidence level. The accuracies for the corrected GPS locations were found to be \pm 2 feet, and in all but a few cases were found to be of a higher accuracy, compared to the digitized locations. In a few cases, the GPS base station data did not contain enough information to get reasonable locations and the digitized values were used.

Vertical control was obtained using a Trimble GeoExplorer 3 handheld GPS unit, American Paulin Model T-5 altimeters and USGS topographic maps at a scale of 1:63,360. The altimetry data was collected using three Paulin altimeters with the readings averaged at each station and corrected for diurnal barometric variations. Where feasible, the gravity stations were located at U.S. Coast and Geodetic Survey Vertical Angle Benchmarks (VABM) for comparison. Temperature and drift corrections were also applied and yielded elevations with an accuracy of \pm 30 feet. Elevations were also digitized from USGS topographic maps with an accuracy of \pm 50 feet and the GPS data were processed as described above and yielded data with an accuracy of \pm 2 feet. A comparison of the three elevations was made (fig. 2) and it was determined that for most of the stations, the GPS values provided the most accurate and consistent values for use in reducing the data.
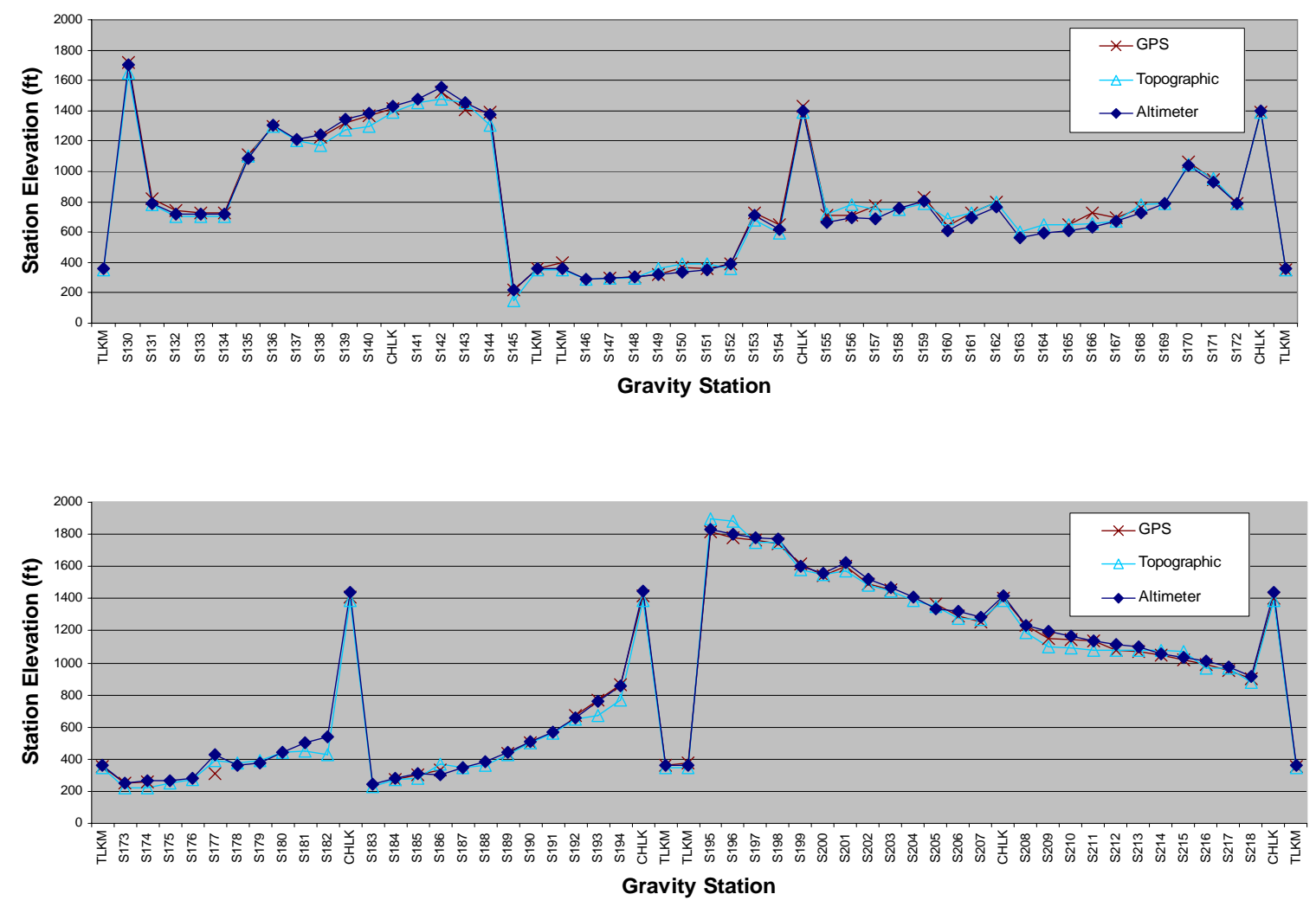

Figure 2. Graph comparing the altimetry, GPS, and topographic station elevations. 
Gravity reductions were run on all of the data (including the data obtained from the USGS) using standard techniques. Corrections for the variation of gravity with latitude at each station were computed based on the Geodetic Reference System 1967 (International Association of Geodesy, 1971) using the International Gravity Standardization Net 1971 gravity datum (Morelli and others, 1974). The observed gravity values were calculated by adding the meter drift and earth-tide corrections to the meter readings converted to milligals. Free-air anomalies were calculated by subtracting the theoretical gravity from the observed gravity and adding a free-air correction. Simple Bouguer anomalies were calculated by subtracting the Bouguer correction from the free-air anomaly, calculated using a gravitational constant of $6.67 \times 10^{-11} \mathrm{~m}^{3}$ $\mathrm{kg}^{-1} \mathrm{~s}^{-2}$ and a standard density of $2.67 \mathrm{gm} / \mathrm{cc}$. Complete Bouguer anomalies were calculated by adding the terrain correction to the simple Bouguer anomaly and isostatic anomalies were calculated by adding the isostatic correction to the complete Bouguer anomaly.

Bob Morin of the USGS computed the terrain corrections for this data by using a computer program (Plouff, 1966, 1977; Godson and Plouff, 1988) and a digital terrain model. This program calculated the gravity effects of the surrounding terrain for each station from a radial distance of $0.39 \mathrm{~km}$ to a distance of $166.7 \mathrm{~km}$ using the standard Hammer technique (Hammer, 1939), in which average elevation estimates within zones surrounding the station are used to compute the gravity effect of each zone. The station elevations used for this correction were taken from the digitized 1:63,360-scale USGS topographic maps in order to be consistent with the elevation model used for the terrain. No inner zone correction was applied due to the flat topography surrounding the stations.

Bob Morin also processed these data with an isostatic reduction program (Jachens and Roberts, 1981) to compensate for the effects of crustal roots that buoyantly support topography. The isostatic reduction assumes an Airy-Heiskanen model with a density of topography above sea level of $2.6 \mathrm{gm} / \mathrm{cc}$ and a crustal thickness at sea level of $25 \mathrm{~km}$.

The principal facts for the gravity stations collected during this survey are listed in table 1. Figure 3 shows the contoured free-air anomaly values, figure 4 shows the contoured complete Bouguer anomaly values, and figure 5 shows the contoured isostatic anomaly values. 
Table 1. Principal facts for the gravity stations collected during this survey. Topo represents the topographic elevation taken from 1:63,360-scale USGS topographic maps. Elev represents the station elevation used for reducing the data. FAA is the free-air anomaly, SBA is the simple Bouguer anomaly, CBA is the complete Bouguer anomaly and IA is the isostatic anomaly. Latitude and longitude are projected in NAD27.

\begin{tabular}{|c|c|c|c|c|c|c|c|c|c|c|c|}
\hline Station & Topo & & Elev & & Lat (NAD27) & Lon (NAD27) & Obs Grav & FAA & SBA & CBA & IA \\
\hline CHLK & 1390.00 & $\mathrm{~F}$ & 1410.00 & $\mathrm{~F}$ & 62.43421893 & -151.40674693 & 981926.584 & -44.078 & -92.167 & -91.027 & -20.417 \\
\hline $\mathrm{S} 130$ & 1650.00 & $\mathrm{~F}$ & 1719.88 & $\mathrm{~F}$ & 62.45262168 & -151.06676121 & 981907.583 & -35.303 & -93.961 & -92.561 & -23.941 \\
\hline $\mathrm{S} 131$ & 785.00 & $\mathrm{~F}$ & 818.64 & $\mathrm{~F}$ & 62.42086911 & -151.10736464 & 981954.951 & -70.341 & -98.262 & -97.072 & -30.942 \\
\hline $\mathrm{S} 132$ & 700.00 & $\mathrm{~F}$ & 745.83 & $\mathrm{~F}$ & 62.40927160 & -151.13960928 & 981955.335 & -75.942 & -101.379 & -100.429 & -34.989 \\
\hline $\mathrm{S} 133$ & 700.00 & $\mathrm{~F}$ & 723.82 & $\mathrm{~F}$ & 62.39511993 & -151.17311482 & 981951.711 & -80.581 & -105.268 & -104.488 & -39.968 \\
\hline $\mathrm{S} 134$ & 700.00 & $\mathrm{~F}$ & 723.74 & $\mathrm{~F}$ & 62.36399494 & -151.18546654 & 981954.804 & -75.175 & -99.859 & -99.169 & -37.139 \\
\hline $\mathrm{S} 135$ & 1100.00 & $\mathrm{~F}$ & 1110.32 & $\mathrm{~F}$ & 62.37694732 & -151.24552902 & 981927.572 & -67.012 & -104.880 & -104.090 & -40.340 \\
\hline S136 & 1300.00 & $\mathrm{~F}$ & 1299.70 & $\mathrm{~F}$ & 62.36115999 & -151.25322324 & 981915.252 & -60.341 & -104.669 & -104.109 & -41.609 \\
\hline S137 & 1200.00 & $\mathrm{~F}$ & 1210.60 & $\mathrm{~F}$ & 62.34217841 & -151.27890160 & 981917.704 & -64.854 & -106.143 & -105.833 & -44.583 \\
\hline $\mathrm{S} 138$ & 1175.00 & $\mathrm{~F}$ & 1226.17 & $\mathrm{~F}$ & 62.32976656 & -151.30645244 & 981915.689 & -64.479 & -106.299 & -105.999 & -45.429 \\
\hline S139 & 1270.00 & $\mathrm{~F}$ & 1323.94 & $\mathrm{~F}$ & 62.32976656 & -151.30645244 & 981907.497 & -63.474 & -108.629 & -108.299 & -47.729 \\
\hline S140 & 1300.00 & $F$ & 1369.74 & $\mathrm{~F}$ & 62.32206471 & -151.38392997 & 981902.130 & -63.958 & -110.675 & -110.325 & -49.495 \\
\hline S141 & 1450.00 & $\mathrm{~F}$ & 1474.23 & $\mathrm{~F}$ & 62.30203150 & -151.40850208 & 981895.893 & -58.872 & -109.152 & -108.562 & -48.932 \\
\hline S142 & 1475.00 & $\mathrm{~F}$ & 1521.76 & $\mathrm{~F}$ & 62.29237929 & -151.43343835 & 981889.161 & -60.411 & -112.313 & -111.813 & -52.593 \\
\hline S143 & 1450.00 & $\mathrm{~F}$ & 1408.48 & $\mathrm{~F}$ & 62.27235073 & -151.45592286 & 981892.348 & -66.383 & -114.421 & -113.751 & -55.631 \\
\hline S144 & 1305.00 & $\mathrm{~F}$ & 1387.54 & $\mathrm{~F}$ & 62.27234508 & -151.47494029 & 981895.617 & -65.083 & -112.407 & -111.837 & -53.527 \\
\hline S145 & 150.00 & $\mathrm{~F}$ & 221.49 & $\mathrm{~F}$ & 62.21347057 & -151.64343631 & 981961.924 & -104.053 & -111.607 & -110.977 & -53.647 \\
\hline S146 & 290.00 & $\mathrm{~F}$ & 290.01 & $\mathrm{~F}$ & 62.15017002 & -151.71702598 & 981953.875 & -100.915 & -110.806 & -110.416 & -53.876 \\
\hline S147 & 295.00 & $\mathrm{~F}$ & 295.52 & $\mathrm{~F}$ & 62.14737173 & -151.75657562 & 981952.339 & -101.722 & -111.801 & -111.091 & -53.591 \\
\hline $\mathrm{S} 148$ & 300.00 & $\mathrm{~F}$ & 307.52 & $\mathrm{~F}$ & 62.14254669 & -151.77255285 & 981950.757 & -101.814 & -112.303 & -111.403 & -53.553 \\
\hline S149 & 360.00 & $\mathrm{~F}$ & 321.59 & $\mathrm{~F}$ & 62.13596050 & -151.78619255 & 981949.617 & -101.137 & -112.105 & -111.335 & -53.235 \\
\hline S150 & 390.00 & $\mathrm{~F}$ & 365.13 & $\mathrm{~F}$ & 62.13851877 & -151.81123113 & 981948.696 & -98.154 & -110.607 & -109.697 & -50.747 \\
\hline S151 & 390.00 & $\mathrm{~F}$ & 357.97 & $\mathrm{~F}$ & 62.13507861 & -151.83965275 & 981948.112 & 154 & -111.363 & -110.113 & -50.333 \\
\hline S152 & 360.00 & $\mathrm{~F}$ & 391.79 & $\mathrm{~F}$ & 62.13544632 & -151.87618785 & 981951.823 & -92.290 & -105.652 & -103.992 & -42.962 \\
\hline S153 & 680.00 & $\mathrm{~F}$ & 725.36 & $\mathrm{~F}$ & 62.11518099 & -151.84305034 & 981926.759 & -84.458 & -109.197 & -108.667 & -49.157 \\
\hline S154 & 590.00 & $\mathrm{~F}$ & 648.44 & $\mathrm{~F}$ & 62.09663903 & -151.81587020 & 981929.818 & -87.243 & -109.359 & -108.849 & -50.509 \\
\hline S155 & 715.00 & $\mathrm{~F}$ & 707.10 & $\mathrm{~F}$ & 62.06626594 & -151.86655772 & 981932.492 & -76.771 & -100.888 & -100.308 & -40.518 \\
\hline S156 & 780.00 & $\mathrm{~F}$ & 713.99 & $\mathrm{~F}$ & 62.06182958 & -151.88222824 & 981933.738 & -74.543 & -98.894 & -98.324 & -37.964 \\
\hline S157 & 750.00 & $\mathrm{~F}$ & 775.07 & $\mathrm{~F}$ & 62.05739415 & -151.90715730 & 981935.518 & -66.684 & -93.119 & -92.219 & -30.949 \\
\hline S158 & 750.00 & $\mathrm{~F}$ & 755.80 & $\mathrm{~F}$ & 62.05456646 & -151.93069628 & 981938.031 & -65.772 & -91.550 & -90.310 & -28.170 \\
\hline S159 & 790.00 & $\mathrm{~F}$ & 830.96 & $\mathrm{~F}$ & 62.05060337 & -151.94500223 & 981937.221 & -59.215 & -87.556 & -86.346 & -23.656 \\
\hline S160 & 690.00 & $\mathrm{~F}$ & 641.34 & $\mathrm{~F}$ & 62.03618646 & -151.934 & 226 & 962 & 836 & 866 & -27.556 \\
\hline S161 & 730.00 & $\mathrm{~F}$ & 726.39 & $\mathrm{~F}$ & 62.01827345 & -151.98308811 & 981946.947 & -56.895 & -81.669 & -79.869 & -15.539 \\
\hline S162 & 800.00 & $\mathrm{~F}$ & 794.92 & $\mathrm{~F}$ & 62.01349530 & -152.02203294 & 981945.520 & -51.517 & -78.628 & -74.118 & -8.168 \\
\hline S163 & 600.00 & $\mathrm{~F}$ & 561.09 & $\mathrm{~F}$ & 61.96314503 & -152.06990560 & 981955.752 & -59.490 & -78.626 & -76.256 & -7.556 \\
\hline S164 & 650.00 & $\mathrm{~F}$ & 590.44 & $\mathrm{~F}$ & 61.95794576 & -152.10610384 & 981952.969 & -59.120 & -79.258 & -76.218 & -5.888 \\
\hline S165 & 650.00 & $\mathrm{~F}$ & 647.05 & $\mathrm{~F}$ & 61.95573624 & -152.12016342 & 981951.727 & -54.873 & -76.941 & -73.591 & -2.641 \\
\hline S166 & 660.00 & $\mathrm{~F}$ & 633.31 & $\mathrm{~F}$ & 61.94937872 & -152.13877316 & 981948.484 & -58.929 & -80.528 & -76.558 & -4.678 \\
\hline S167 & 670.00 & $\mathrm{~F}$ & 692.66 & $\mathrm{~F}$ & 61.95403293 & -152.18652940 & 981949.048 & -53.132 & -76.756 & -72.396 & 1.334 \\
\hline S168 & 780.00 & $F$ & 759.25 & $\mathrm{~F}$ & 61.95528988 & -152.21908736 & 981947.767 & -48.245 & -74.140 & -69.940 & 5.030 \\
\hline
\end{tabular}


Table 1 (continued)

\begin{tabular}{|c|c|c|c|c|c|c|c|c|c|c|c|}
\hline Station & Topo & & Elev & & Lat (NAD27) & Lon (NAD27) & Obs Grav & FAA & SBA & CBA & IA \\
\hline S169 & 790.00 & $\mathrm{~F}$ & 788.33 & $\mathrm{~F}$ & 61.94662479 & -152.21565530 & 981939.565 & -53.059 & -79.946 & -75.356 & -0.276 \\
\hline S170 & 1050.00 & $\mathrm{~F}$ & 1062.38 & $\mathrm{~F}$ & 61.92804658 & -152.24040590 & 981919.031 & -46.416 & -82.650 & -72.080 & 4.460 \\
\hline S171 & 950.00 & $F$ & 946.47 & $\mathrm{~F}$ & 61.99078254 & -152.05591686 & 981938.238 & -42.835 & -75.116 & -73.116 & -5.526 \\
\hline S172 & 790.00 & $\mathrm{~F}$ & 790.61 & $\mathrm{~F}$ & 62.00531692 & -152.03938853 & 981945.565 & -51.262 & -78.226 & -73.806 & -7.086 \\
\hline S173 & 220.00 & $\mathrm{~F}$ & 254.43 & $\mathrm{~F}$ & 62.29702321 & -151.77379266 & 981989.802 & -79.323 & -88.000 & -86.630 & -22.170 \\
\hline S174 & 220.00 & $\mathrm{~F}$ & 256.88 & $\mathrm{~F}$ & 62.31447064 & -151.79448801 & 981993.581 & -76.617 & -85.378 & -83.668 & -17.718 \\
\hline S175 & 250.00 & $\mathrm{~F}$ & 268.48 & $\mathrm{~F}$ & 62.34201711 & -151.81407498 & 981996.598 & -74.564 & -83.721 & -80.921 & -12.881 \\
\hline S176 & 270.00 & $\mathrm{~F}$ & 282.92 & $\mathrm{~F}$ & 62.34848582 & -151.85405913 & 981994.315 & -75.972 & -85.622 & -83.432 & -14.222 \\
\hline S177 & 390.00 & $\mathrm{~F}$ & 428.39 & $\mathrm{~F}$ & 62.37658658 & -151.88153455 & 981991.415 & -67.284 & -81.895 & -79.385 & -7.885 \\
\hline S178 & 380.00 & $\mathrm{~F}$ & 360.87 & $\mathrm{~F}$ & 62.39805495 & -151.91057479 & 981997.897 & -68.753 & -81.061 & -78.381 & -4.931 \\
\hline S179 & 390.00 & $\mathrm{~F}$ & 376.68 & $\mathrm{~F}$ & 62.41516118 & -151.91659098 & 981998.167 & -68.270 & -81.118 & -78.028 & -3.318 \\
\hline S180 & 440.00 & $\mathrm{~F}$ & 442.09 & $\mathrm{~F}$ & 62.43262666 & -151.92540345 & 981997.492 & -64.094 & -79.172 & -75.832 & 0.198 \\
\hline S181 & 450.00 & $\mathrm{~F}$ & 499.62 & $\mathrm{~F}$ & 62.44531871 & -151.92425765 & 981996.121 & -60.998 & -78.038 & -74.368 & 2.532 \\
\hline S182 & 430.00 & $\mathrm{~F}$ & 537.03 & $\mathrm{~F}$ & 62.47060277 & -151.93672530 & 981993.158 & -62.323 & -80.639 & -75.929 & 2.921 \\
\hline S183 & 230.00 & $\mathrm{~F}$ & 242.23 & $\mathrm{~F}$ & 62.28231139 & -151.83981262 & 981992.400 & -76.773 & -85.034 & -83.534 & -18.384 \\
\hline S184 & 270.00 & $\mathrm{~F}$ & 272.78 & $\mathrm{~F}$ & 62.30780678 & -151.91325753 & 5.515 & -72.690 & -81 & -79.903 & -11.643 \\
\hline S185 & 280.00 & $\mathrm{~F}$ & 300.60 & $\mathrm{~F}$ & 62.32630397 & -151.96437500 & 981996.097 & -70.871 & -81.123 & -78.513 & -8.013 \\
\hline S186 & 370.00 & $\mathrm{~F}$ & 335.51 & $\mathrm{~F}$ & 62.34416288 & -151.97239785 & 981999.434 & -65.583 & -77.026 & -74.816 & -3.226 \\
\hline S187 & 350.00 & $\mathrm{~F}$ & 347.62 & $\mathrm{~F}$ & 62.37043426 & -152.01813289 & 981998.661 & -67.177 & -79.033 & -76.343 & -2.283 \\
\hline S188 & 360.00 & $\mathrm{~F}$ & 383.52 & $\mathrm{~F}$ & 62.38612727 & -152.07033662 & 981997.975 & -65.655 & -78.736 & -74.816 & 1.264 \\
\hline S189 & 430.00 & $\mathrm{~F}$ & 432.17 & $\mathrm{~F}$ & 62.39990861 & -152.13245792 & 981995.340 & -64.741 & -79.481 & -73.211 & 4.969 \\
\hline S190 & 5 & $\mathrm{~F}$ & 45 & $\mathrm{~F}$ & 7 & 43 & 3 & -6 & -7 & 10 & 00 \\
\hline S191 & 560.00 & $\mathrm{~F}$ & 566.68 & $\mathrm{~F}$ & 62.43562694 & -152.25906704 & 981993.188 & -56.902 & -76.229 & -67.429 & 14.911 \\
\hline S192 & 650.00 & $\mathrm{~F}$ & 671.52 & $\mathrm{~F}$ & 62.45401831 & -152.31163894 & 981993.581 & -48.017 & -70.920 & -60.330 & 23.540 \\
\hline S193 & 670.00 & $\mathrm{~F}$ & 765.28 & $\mathrm{~F}$ & 62.47604094 & -152.36368441 & 981990.680 & -43.738 & -69.838 & -58.038 & 27.182 \\
\hline S194 & 770.00 & $\mathrm{~F}$ & 861.99 & $\mathrm{~F}$ & 62.50205302 & -152.41874189 & 981986.750 & -40.505 & -69.904 & -56.474 & 29.806 \\
\hline S195 & 1900.00 & $\mathrm{~F}$ & 1819.13 & $\mathrm{~F}$ & 742 & 985 & 363 & -2 & 317 & 277 & -1.067 \\
\hline S196 & 1880.00 & $\mathrm{~F}$ & 1775.04 & $\mathrm{~F}$ & 731 & -151.6 & 07 & 55 & -81.795 & -78.075 & -1.075 \\
\hline S197 & 1750.00 & $\mathrm{~F}$ & 1764.61 & $\mathrm{~F}$ & 62.47154929 & -151.58209373 & 981919.109 & -20.978 & -81.161 & -77.601 & -1.891 \\
\hline S198 & 1750.00 & $\mathrm{~F}$ & 1738.05 & $\mathrm{~F}$ & 62.46225940 & -151.56247207 & 981921.568 & -20.325 & -79.603 & -77.073 & -2.353 \\
\hline S199 & 1580.00 & $\mathrm{~F}$ & 1616.79 & $\mathrm{~F}$ & 62.43911310 & -151.49867568 & 981923.973 & -27.603 & -82.746 & -81.386 & -9.416 \\
\hline S200 & 1550.00 & $\mathrm{~F}$ & 1541.29 & $\mathrm{~F}$ & 62.42 & -151.4 & 803 & -37.742 & -90.309 & 359 & -19.099 \\
\hline S201 & 1570.00 & $\mathrm{~F}$ & 1601.07 & $\mathrm{~F}$ & 62.43796500 & -151.49185737 & 981923.265 & -29.704 & -84.310 & -83.010 & -11.220 \\
\hline S202 & 1480.00 & $\mathrm{~F}$ & 1488.68 & $\mathrm{~F}$ & 62.40566100 & -151.44129172 & 981921.891 & -39.244 & -90.017 & -89.227 & -20.877 \\
\hline S203 & 1450.00 & $\mathrm{~F}$ & 1451.99 & $\mathrm{~F}$ & 62.39743510 & -151.41941892 & 981916.007 & -47.966 & -97.488 & -96.808 & -29.418 \\
\hline S204 & 1390.00 & $\mathrm{~F}$ & 1409.16 & $\mathrm{~F}$ & 62.39368102 & -151.38601192 & 981914.151 & -53.571 & -101.632 & -101.032 & -34.342 \\
\hline S205 & 1350.00 & $\mathrm{~F}$ & 1365.68 & $\mathrm{~F}$ & 62.38730335 & -151.35931772 & 981914.181 & -57.156 & -103.734 & -103.194 & -37.334 \\
\hline S206 & 1280.00 & $\mathrm{~F}$ & 1291.82 & $\mathrm{~F}$ & 62.37687273 & -151.32386196 & 981915.585 & -61.922 & -105.981 & -105.501 & -40.941 \\
\hline S207 & 1270.00 & $F$ & 1256.84 & $\mathrm{~F}$ & 62.36867161 & -151.30089159 & 981916.265 & -63.920 & -106.786 & -106.356 & -42.736 \\
\hline S208 & 1190.00 & $\mathrm{~F}$ & 1232.68 & $\mathrm{~F}$ & 62.3566 & -151.29293181 & 981916 & -64.991 & -107.033 & -106.623 & -44.063 \\
\hline S209 & 1100.00 & $\mathrm{~F}$ & 1153.99 & $\mathrm{~F}$ & 62.32409325 & -151.26568815 & 981922.881 & -63.653 & -103.011 & -102.721 & -42.981 \\
\hline $\mathrm{S} 210$ & 1090.00 & $\mathrm{~F}$ & 1140.81 & $\mathrm{~F}$ & 62.31095074 & -151.23891688 & 981929.163 & -57.629 & -96.538 & -96.308 & -37.798 \\
\hline $\mathrm{S} 211$ & 1080.00 & $\mathrm{~F}$ & 1134.88 & $\mathrm{~F}$ & 62.30022854 & -151.20672648 & 981940.864 & -45.686 & -84.392 & -84.132 & -26.702 \\
\hline S212 & 1080.00 & $\mathrm{~F}$ & 1074.35 & $\mathrm{~F}$ & 62.28675723 & -151.19237978 & 981947.931 & -43.305 & -79.947 & -79.717 & -23.357 \\
\hline S213 & 1080.00 & $\mathrm{~F}$ & 1072.66 & $\mathrm{~F}$ & 62.27107748 & -151.18690913 & 981948.274 & -41.950 & -78.534 & -78.344 & -23.074 \\
\hline
\end{tabular}


Table 1 (continued)

\begin{tabular}{|c|c|c|c|c|c|c|c|c|c|c|}
\hline Station & Topo & Elev & & AD27) & NAD27) & Obs & FAA & SBA & CBA & IA \\
\hline S214 & $.00 \mathrm{~F}$ & 23 & $\mathrm{~F}$ & 62.25755016 & -151.15727040 & 981947.900 & -43.329 & -79.182 & 2 & 02 \\
\hline S215 & $1070.00 \mathrm{~F}$ & 1017.58 & $\mathrm{~F}$ & 62.23843536 & -151.12845477 & 981956.187 & -36.776 & 482 & -71.282 & -18.572 \\
\hline S216 & 970.00 & 985.49 & $\mathrm{~F}$ & 62. & 133 & 98 & 15 & 6 & 66 & -27.236 \\
\hline S217 & $970.00 \quad \mathrm{~F}$ & 953.65 & $\mathrm{~F}$ & 62.19109889 & -151.07827869 & 981941.538 & -53.896 & -86.421 & -86.121 & -36.541 \\
\hline $\mathrm{S} 218$ & 880.00 & 903.95 & $\mathrm{~F}$ & 62.16995903 & -151.05426283 & 981940.080 & -58.445 & -89.275 & -88.975 & -40.765 \\
\hline
\end{tabular}




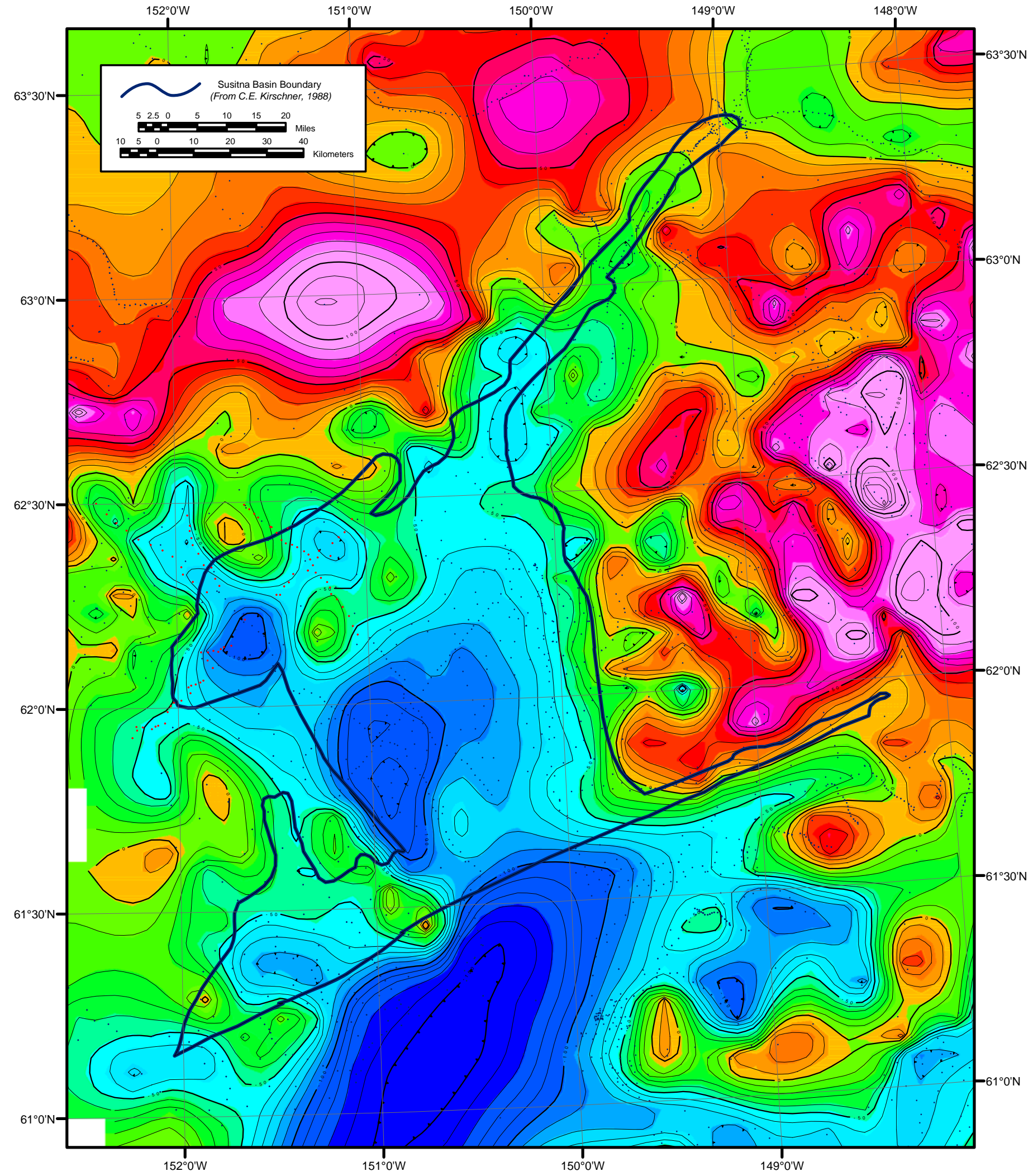

Figure 3. Free-air gravity map of the Susitna basin with a contour interval of $10 \mathrm{mGal}$. 


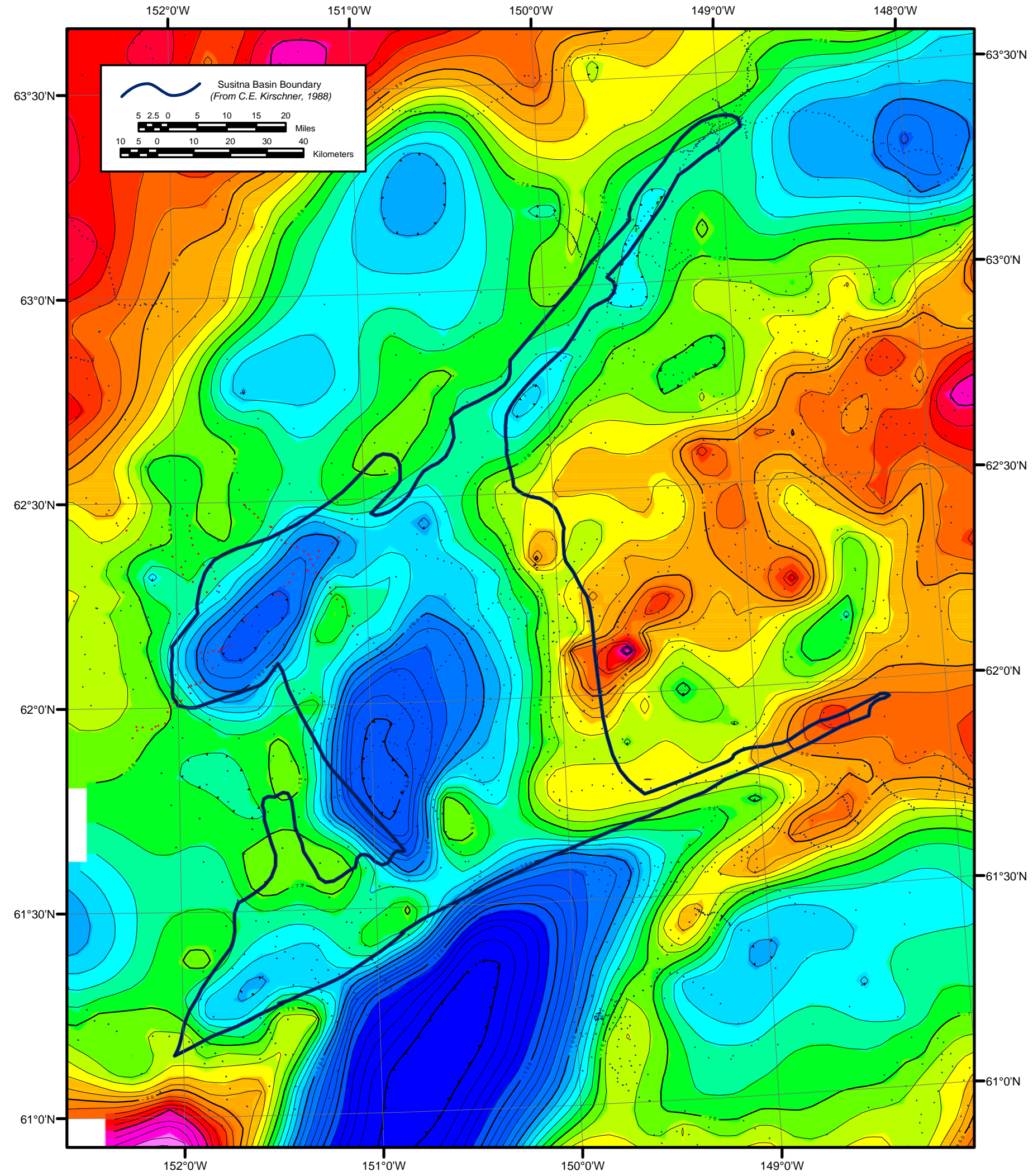

Figure 4. Complete Bouguer gravity map of the Susitna basin with a contour interval of $5 \mathrm{mGal}$. 


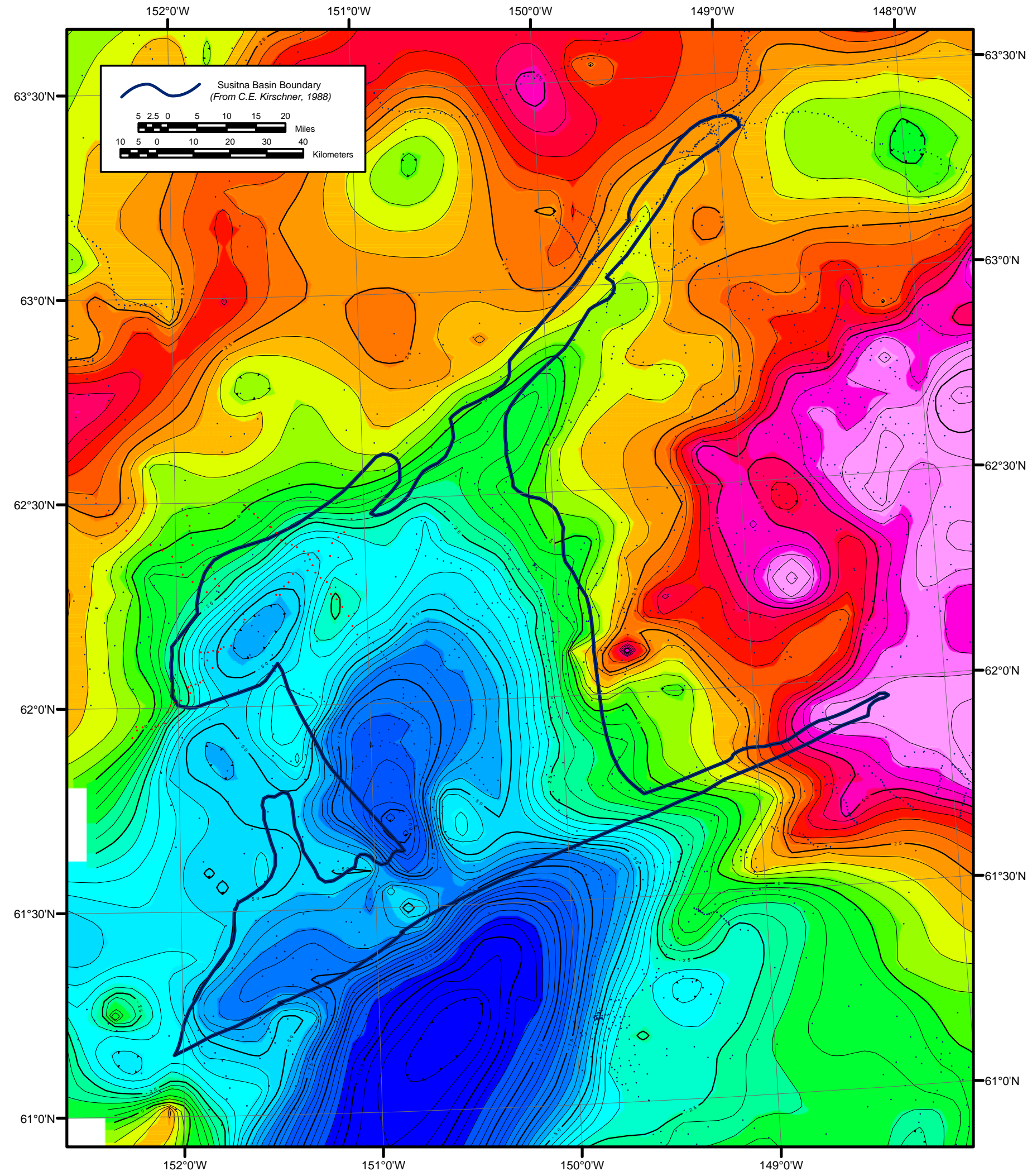

Figure 5. Isostatic gravity map of the Susitna basin with a contour interval of $5 \mathrm{mGal}$. 


\section{ACKNOWLEDGMENTS}

The author gratefully acknowledges Ken Helmold, Don Krouskop, and Tim Ryherd of the Division of Oil \& Gas for reviewing the manuscript and providing many helpful suggestions. I also want to thank Kristin Dirks and Christy Beaty with the Division of Oil \& Gas for their assistance in collecting and reducing this data, and Bob Morin with the USGS for computing all of the terrain and isostatic corrections.

\section{REFERENCES CITED}

Barnes, D.F., 1968, Alaska gravity base station network: U.S. Geological Survey Open-File Report 68-4, $34 \mathrm{p}$.

1972, Southeast Alaska gravity base station network: U.S. Geological Survey Open-File Report 72-18, 40 p.

1977, Bouguer gravity map of Alaska: U.S. Geological Survey Geophysical Investigations Map GP-913, 1 sheet, scale 1:2,500,000.

Godson, R.H., and Plouff, D., 1988, BOUGUER Version 1.0, A microcomputer gravity-terrain-correction program: U.S. Geological Survey Open-File Report 88-644-A, Documentation, 22 p.; 88-644-C, $5 \frac{1}{4}$ inch diskette.

Hammer, S., 1939, Terrain corrections for gravimeter stations: Geophysics, v. 4, p. 184-194.

International Association of Geodesy, 1971, Geodetic reference system, 1967: Paris, Bureau Central de l'Association Internationale de Geodesie, Special Publication 3, 116 p.

Jachens, R.C., and Roberts, C.W., 1981, Documentation of a FORTRAN program, 'isocomp', for computing isostatic residual gravity: U.S. Geological Survey Open-File Report 81-574, 26 p.

Kirschner, C.E., 1988, Map showing sedimentary basins of onshore and continental shelf areas, Alaska: U.S. Geological Survey Miscellaneous Investigations Series Map I-1873, 1 sheet, scale $1: 2,500,000$.

Maynard, D.L., 1987, Water-well data from the Houston area, Matanuska-Susitna Borough, Alaska: Alaska Division of Geological \& Geophysical Surveys, Report of Investigations 87-17, 14 p., 1 sheet, scale 1:25,000.

Meyer, J.F., Jr, and Boggess, P.L., 2003, Principal facts for gravity data collected in the Susitna Basin area, southcentral Alaska: Alaska Division of Geological \& Geophysical Surveys, Preliminary Interpretive Report 2003-3, 13 p.

Morelli, C., Gantar, C., Honkasala, T., McConnell, R.K., Tanner, J.G., Szabo, B., Uotila, U.A., and Whalen, C.T., 1974, The International Gravity Standardization Net 1971 (IGSN 71): Paris, Bureau Central de l’Association Internationale de Geodesie, Special Publication 4, 194 p.

Plouff, D., 1966, Digital terrain corrections based on geographic coordinates [abs.]: Geophysics, v.31, no. 6, p. 1208.

1977, Preliminary documentation for a Fortran program to compute gravity terrain corrections based on topography digitized on a geographic grid: U.S. Geological Survey Open-File Report 77535, $45 \mathrm{p}$.

Selkregg, L.L., 1974, Alaska regional profiles: southcentral region, Vol 1: Arctic Environmental Information and Data Center, University of Alaska, 255 p.

Turner, D.L., and Wescott, E.M., 1982, A Preliminary Investigation of the Geothermal Energy Resources of the Lower Susitna Basin: University of Alaska Geophysical Institute Report UAG R-287, 50 p. 